\title{
Maharam Spectra of Loeb Spaces ${ }^{1}$
}

\author{
Renling Jin ${ }^{2} \&$ H. Jerome Keisler ${ }^{3}$
}

\begin{abstract}
We characterize Maharam spectra of Loeb probability spaces and give some applications of the results.
\end{abstract}

\section{Introduction}

In the nonstandard approach to probability theory, a central role is played by a family of very rich probability spaces, known as Loeb spaces. It is natural to ask for a description of the Loeb spaces, or at least a description of their measure algebras, in standard terms. By Maharam's Theorem (see $\S 1$ ), the measure algebra of any atomless probability space $(\Omega, \mathcal{B}, \nu)$ is determined up to isomorphism by a finite or countable set of "weighted" infinite cardinals, which we will call the Maharam spectrum of $(\Omega, \mathcal{B}, \nu)$.

In this paper we will study the Maharam spectra of Loeb probability spaces. We will concentrate on the two classes of Loeb spaces which are most frequently used in applications: the hyperfinite Loeb spaces and the Loeb spaces generated by standard probability spaces. In general, the possible Maharam spectra for these Loeb spaces will depend on the nonstandard universe in which one is working.

We will prove the following three theorems. If $X$ is a set, $\operatorname{card}(X)$ is the size of $X$ in the usual set-theoretic sense; if $r \in{ }^{*} \mathbb{R}, \operatorname{card}(r)$ denotes $\operatorname{card}\left({ }^{*} \mathbb{N} \cap(0, r]\right)$.

A. (See Theorem 2.1). Let $(\Omega, \mathcal{A}, \mu)$ be a hyperfinite set with the normalized counting probability measure. Then the corresponding Loeb space has Maharam spectrum $\left\{\operatorname{card}\left(2^{|\Omega|}\right)\right\}$.

B. (See Theorem 3.2). Let $(\Omega, \mathcal{A}, \mu)$ be a hyperfinite internal probability space where each point $x \in \Omega$ has infinitesimal weight $w(x)$, and let $\kappa(x)=\operatorname{card}\left(2^{1 / w(x)}\right)$. Then the Maharam spectrum of the corresponding Loeb space is the set of all cardinals $\lambda$ such that the set $\{x \in \Omega: \kappa(x)=\lambda\}$ has positive Loeb measure.

\footnotetext{
${ }^{1}$ Mathematics Subject Classification Primary 03H05, 28E05. Secondary 03G05

${ }^{2}$ The research of the first author was supported by NSF postdoctoral fellowship \#DMS-9508887.

${ }^{3}$ The research of the second author was partially supported by an NSF grant and the Vilas Trust Fund.
} 
C. (See Theorem 4.2). Let $(\Omega, \mathcal{B}, \nu)$ be a standard atomless probability space with Maharam spectrum $S$. Then the Maharam spectrum of the Loeb space generated by $\left({ }^{*} \Omega,{ }^{*} \mathcal{B},{ }^{*} \nu\right)$ is the set $\left\{\operatorname{card}\left({ }^{*} \kappa\right): \kappa \in S\right\}$.

In [7] and [9] Maharam's Theorem is successfully used in studying the compactness of Loeb spaces and the effects of strong saturation properties of nonstandard universes on Loeb spaces. It is shown in [7] that the Maharam spectrum of a hyperfinite Loeb space with the normalized counting probability measure is a singleton $\{\lambda\}$ for some $\lambda$. This allows one to translate some problems about these Loeb spaces to problems about the simplest classical measure algebras. Our first theorem in this paper pinpoints $\lambda$ in terms of the cardinality of the Loeb space. Sometimes we need to know the value of $\lambda$; for example, see Corollary 2.5. In general the Maharam spectrum of a Loeb space may have more than one cardinal. A clear characterization of the Maharam spectrum of a Loeb space will give us a better understanding of the Loeb measure construction and may be helpful in deriving sharper applications of Maharam's Theorem.

We introduce notation in $\S 1$. In $\S 2$ we prove Theorem A above and give an application. In $\S 3$ we prove Theorem B above. It will follow that the nonstandard universe can be chosen so that any prescribed finite or countable set of distinct infinite cardinals, with some obvious restrictions, can be the Maharam spectrum of a hyperfinite Loeb probability space. In $\S 4$ we prove Theorem $\mathrm{C}$ above. In $\S 5$ we explore the possibility of representing the Maharam spectrum of a Loeb space $(\Omega, L(\mathcal{A}), L(\mu))$ as the Maharam spectrum of the Loeb space generated by a hyperfinite subalgebra $\mathcal{B} \subseteq \mathcal{A}$. In $\S 6$ we point out that the nonstandard universe can be chosen so that the Maharam spectra of Loeb spaces are extremely simple.

\section{Preliminaries}

We write $\kappa, \lambda, \tau, \ldots$ for infinite cardinals, and write $\alpha, \beta, \gamma, \ldots$ for ordinals.

We begin with preliminary material in measure theory. For simplicity we consider only finite countably additive measure spaces $(\Omega, \mathcal{B}, \nu)$ in this paper. By a measure algebra isomorphism between two such spaces we will mean a bijection between the associated measure algebras which preserves Boolean operations and measures. A collection $X$ of sets completely generates $\mathcal{B}$ if $\mathcal{B}$ is contained in the $\nu$-completion of the $\sigma$-algebra generated by $X$. 
For each $A \in \mathcal{B}$, let $(\Omega, \mathcal{B}, \nu)\lceil A$ be the measure space $(A, \mathcal{B} \cap \mathcal{P}(\mathcal{A}), \nu)$ formed by restricting $(\Omega, \mathcal{B}, \nu)$ to $A$. We denote by $\left(\{0,1\}^{\lambda}\right)$ the product of $\lambda$ copies of the two-point probability space $\{0,1\}$ where each point has measure $\frac{1}{2}$.

Maharam's Theorem (cf. Theorem 3.9 of [2]) may be stated as follows.

Given an atomless probability space $(\Omega, \mathcal{B}, \nu)$, there is a finite or countable set of distinct infinite cardinals $\left\{\lambda_{i}: i \in I\right\}$, and a partition $\left\{C_{i}: i \in I\right\}$ of $\Omega$ with each $C_{i}$ having positive measure $r_{i}=\nu\left(C_{i}\right)$, such that for each $i \in I,(\Omega, \mathcal{B}, \nu) \uparrow C_{i}$ is measure algebra isomorphic to $r_{i} \cdot\left(\{0,1\}^{\lambda_{i}}\right)$.

It follows that the partition $\left\{C_{i}: i \in I\right\}$ is unique up to a null set, and the set of pairs $\left(r_{i}, \lambda_{i}\right)$ is unique and determines the measure algebra of $(\Omega, \mathcal{B}, \nu)$ up to isomorphism. We will call $\left\{C_{i}: i \in I\right\}$ a Maharam partition of $(\Omega, \mathcal{B}, \nu)$, and we define the Maharam spectrum of $(\Omega, \mathcal{B}, \nu)$ to be the set of cardinals $\left\{\lambda_{i}: i \in I\right\}$.

The Maharam type of $(\Omega, \mathcal{B}, \nu)$ is the least cardinal $\lambda$ such that $\mathcal{B}$ is completely generated by a set of cardinality $\lambda$. For each $A \in \mathcal{B}$ we will let $\tau(A)$ denote the Maharam type of the restriction $(\Omega, \mathcal{B}, \nu)\lceil A$, with the convention that $\tau(A)=0$ if $\nu(A)=0$. In particular, $\tau(\Omega)$ is the Maharam type of the whole space $(\Omega, \mathcal{B}, \nu)$. $(\Omega, \mathcal{B}, \nu)$ is called homogeneous if and only if $\tau(A)=\tau(\Omega)$ for every $A \in \mathcal{B}$ of positive measure. One can see from Maharam's theorem that the Maharam type is equal to the supremum of the Maharam spectrum.

We will use the following consequence of Maharam's theorem.

An atomless probability space is measure algebra isomorphic to $\left(\{0,1\}^{\lambda}\right)$ if and only if the Maharam spectrum is the singleton set $\{\lambda\}$, and also if and only if the space is homogeneous and has Maharam type $\lambda$.

We now turn to preliminaries in nonstandard analysis. Let $\mathbb{N}$ be the set of all standard non-negative integers and let $\mathbb{R} \supseteq \mathbb{N}$ be the set of all standard real numbers. Let $Z$ be a set containing $\mathbb{R}$ and all standard objects under consideration. Starting from $Z$ considered as a set of urelements, we construct the standard superstructure $V=V(Z)=\bigcup_{n \in \mathbb{N}} V_{n}$, where $V_{0}=Z$ and $V_{n+1}=V_{n} \cup \mathcal{P}\left(V_{n}\right)$ for every $n \in \mathbb{N}$, together with the membership relation $\in$. By a nonstandard universe, denoted by $\left({ }^{*} V, \in\right)$, we mean the Mostowski collapse of the truncation at rank $\omega$ of an elementary extension of $(V, \in)$ such that the image ${ }^{*} \mathbb{N}$ of $\mathbb{N}$ properly contains $\mathbb{N}$. For brevity we often write ${ }^{*} V$ instead of $\left({ }^{*} V, \in\right)$. Let $*: V \mapsto{ }^{*} V$ be the natural embedding. Thus 
${ }^{*} V$ is a transitive subset of a second superstructure $V\left({ }^{*} Z\right)$. A set $A \in V\left({ }^{*} Z\right)$ is called internal (in ${ }^{*} V$ ) if $A$ is an element of ${ }^{*} V$, and is called external otherwise. If $X$ is internal in ${ }^{*} V$ we denote by $|X|$ the internal cardinality of $X$ in ${ }^{*} V$. The reader may consult $\S 4.4$ of [1], [14] or [4] for more information on nonstandard analysis and nonstandard universes. In this paper let's fix, unless otherwise specified, an at least $\omega_{1}$-saturated nonstandard universe ${ }^{*} V$.

For $x, y \in{ }^{*} \mathbb{R}, x \approx y$ means $x-y$ is infinitesimal. We write $s t(x)=r$ iff $r \in \mathbb{R}$ and $x \approx r$. Elements of ${ }^{*} \mathbb{N}$ are called hyperintegers. A set $X$ is said to by hyperfinite if $|X|$ is an infinite hyperinteger, i.e. $|X| \in{ }^{*} \mathbb{N} \backslash \mathbb{N}$. For any $x, y \in{ }^{*} \mathbb{N}$ we write $[x, y)$ exclusively for an interval $\{x, x+1, \ldots, y-1\}$ in ${ }^{*} \mathbb{N}$ rather than an interval of reals. The symmetric difference of two sets $A$ and $B$ is denoted by $A \Delta B$.

By an internal measure space we mean an internal object $(\Omega, \mathcal{A}, \mu) \in{ }^{*} V$ which satisfies the formula defining a (countably additive) measure space in the model ${ }^{*} V$. By a standard measure space we mean a (usually external) object in either $V(Z)$ or $V\left({ }^{*} Z\right)$ which is a measure space in the ordinary sense. We use a similar convention for other properties. Given an internal measure space $(\Omega, \mathcal{A}, \mu)$, with $\mu(\Omega)$ finite, one can form a real-valued finitely additive measure space $(\Omega, \mathcal{A}, s t \circ \mu)$. Then by the Loeb construction one can extend $\mathcal{A}$ to a smallest complete $\sigma$-algebra $L(\mathcal{A})$ and extend st $\circ \mu$ to a measure $L(\mu)$ on $L(\mathcal{A})$ such that $(\Omega, L(\mathcal{A}), L(\mu))$ is a complete countably additive measure space with $L(\mu)(\Omega)=s t(\mu(\Omega))<+\infty$. The space $(\Omega, L(\mathcal{A}), L(\mu))$ is called the Loeb space generated by the internal space $(\Omega, \mathcal{A}, \mu)$. Loeb spaces are important tools in applying nonstandard methods to probability theory. See [14], [18] or [19] for more information on the construction of Loeb spaces. Among all Loeb spaces, two classes are most frequently used. One is the class of all hyperfinite Loeb spaces and the other is the class of all Loeb spaces generated by standard probability spaces. All internal measure spaces are understood to be ${ }^{*}$ countably additive in this paper (although the Loeb construction works even when one starts with an internal finitely additive measure space.)

A Loeb space $(\Omega, L(\mathcal{A}), L(\mu))$ is called hyperfinite iff $|\Omega|$ is hyperfinite and $\mathcal{A}=$ ${ }^{*} \mathcal{P}(\Omega)$ (the set of all internal subsets of $\Omega$ ). If, in addition, $\mu$ is the normalized counting probability measure $\mu(A)=|A| /|\Omega|$ for all $A \in \mathcal{A}$, then $(\Omega, L(\mathcal{A}), L(\mu))$ is called the uniform hyperfinite Loeb space on $\Omega$.

Given a standard measure space $(\Omega, \mathcal{B}, \nu)$ in the original superstructure $V(Z)$ with $\nu(\Omega)$ finite, the counterpart $\left({ }^{*} \Omega,{ }^{*} \mathcal{B},{ }^{*} \nu\right)$ is an internal measure space. The cor- 
responding Loeb space $\left({ }^{*} \Omega, L\left({ }^{*} \mathcal{B}\right), L\left({ }^{*} \nu\right)\right)$ is called the Loeb space generated by the standard measure space $(\Omega, \mathcal{B}, \nu)$.

\section{Uniform hyperfinite Loeb spaces}

We state the main theorem in this section first.

Theorem 2.1 The Maharam spectrum of a uniform hyperfinite Loeb probability space $(\Omega, L(\mathcal{A}), L(\mu))$ is $\left\{\operatorname{card}\left(2^{|\Omega|}\right)\right\}$.

Here $|\Omega|$ is the internal cardinality of $\Omega$, which is an infinite hyperinteger. Thus the exponent $2^{|\Omega|}$ is the internal cardinality of the set of all internal subsets of $\Omega$, and $\operatorname{card}\left(2^{|\Omega|}\right)$ is the external cardinality of this set. The proof of the theorem needs the following two lemmas.

Lemma 2.2 Any uniform hyperfinite Loeb probability space $(\Omega, L(\mathcal{A}), L(\mu))$ is homogeneous.

Proof: The lemma is proved in [7]. We would like to include the proof here for self-containment.

Let

$$
\kappa=\min \{\tau(C): L(\mu)(C)>0\} .
$$

It suffices to show that $\tau(\Omega)=\kappa$. Since $(\Omega, L(\mathcal{A}), L(\mu))$ is atomless, $\kappa$ is infinite. Let $C \in L(\mathcal{A})$ be such that $\tau(C)=\kappa$. Then $L(\mu)(C)>0$, so there is an internal set $A \subseteq C$ such that $L(\mu)(A)>0$. Thus $m=[H / K]$ is finite, where $H=|\Omega|$ and $K=|A|$. For each $i=0,1, \ldots, m-1$, let $A_{i}=[i \cdot K,(i+1) \cdot K)$ and let $A_{*}=[m \cdot K, H)$. Then $\left|A_{i}\right|=K$ for each $i<m$, and $\left|A_{*}\right| \leqslant K$. Therefore $\tau\left(A_{i}\right)=\kappa$ for each $i<m$, and $\tau\left(A_{*}\right) \in\{0, \kappa\}$. Hence

$$
\kappa \leqslant \tau(\Omega) \leqslant\left(\prod_{i=0}^{k-1} \tau\left(A_{i}\right)\right) \cdot \tau\left(A_{*}\right) \leqslant \kappa^{k+1}=\kappa .
$$

The next lemma is a purely finite combinatorial result. Given $n \in \mathbb{N}$, let $\Omega_{n}=$ $[0, n)$ and let $\mu_{n}$ be the normalized counting measure on $\Omega_{n}$, i.e. $\mu_{n}(A)=|A| / n$ for any $A \subseteq \Omega_{n}$. Given a $k \in \mathbb{N}$ and a $c \in \mathbb{R}$ with $c>1$, we call a sequence $\left\langle A_{i}: i<J\right\rangle$ of 
subsets of $\Omega_{n}$ a $(k, c)$-independent sequence iff for any $\sigma \subseteq[0, J)$ with $|\sigma| \leqslant k$ and for any $h \in 2^{\sigma}$, we have

$$
\mu_{n}\left(\bigcap_{i \in \sigma} A_{i}^{h(i)}\right)>\frac{1}{2^{|\sigma|} \cdot c}
$$

where we write $A^{0}=A$ and $A^{1}=\Omega_{n} \backslash A$ for every $A \subseteq \Omega_{n}$. Note that if $k=\infty$ and $c=1$ then a $(k, c)$-independent sequence is an independent sequence of measurable sets with measure $\frac{1}{2}$.

Lemma 2.3 For every $k \in \mathbb{N}$ and for every $c \in \mathbb{R}$ with $c>1$ there is a $K \in \mathbb{N}$ such that for all large enough $n$ there exists a $(k, c)$-independent sequence of length $\left[2^{n / K}\right]$ on $\Omega_{n}$.

Proof: The idea of the following probabilistic argument is due to S. Shelah (see Fact 3 in $\S 1$ of [9]). Let's fix $k$ and $c$. Let $J>k$ and consider a $J \times n$-array of independent coin-tossing experiments with respect to a probability measure $P$. For each $i<J$ let $A_{i} \subseteq \Omega_{n}$ be such that $m \in A_{i}$ iff the outcome of $(m, i)$-th coin-tossing is a head. Given any $\sigma \subseteq[0, J)$ with $|\sigma|=s \leqslant k$, and any function $h \in 2^{\sigma}$, let $p=2^{-s}$ and $A^{h}=\bigcap_{i \in \sigma} A_{i}^{h(i)}$. Then $\left\langle A_{i}, i<J\right\rangle$ is a $(k, c)$-independent sequence if and only if $\mu_{n}\left(A^{h}\right)>p / c$ for every $\sigma$ and $h$. Our plan is to get an upper bound for the probability of the event $\mu_{n}\left(A^{h}\right) \leqslant p / c$ and use this upper bound to show that with positive probability none of these events occur.

Fix $\sigma$ with $|\sigma|=s$ and $h \in 2^{\sigma}$. For each $m<n$, let $X_{m}=1-p$ if $m \in A^{h}$ and $X_{m}=-p$ otherwise. The $X_{m}$ are independent random variables with mean zero, and their sum is

$$
X=\sum_{m<n} X_{m}=n \cdot\left(\mu_{n}\left(A^{h}\right)-p\right) .
$$

Thus $\mu_{n}\left(A^{h}\right) \leqslant p / c$ if and only if $X \leqslant-q \cdot n$ where $q=p \cdot\left(1-c^{-1}\right)$. We can get the desired bound from Bernstein's inequality (see [10], Lemma 3.1), which says that whenever $z \leqslant \sqrt{n} / 4$,

$$
P[|X| \geqslant z \sqrt{n}] \leqslant 2 e^{-z^{2} / 4}
$$

We can assume without loss of generality that $c \leqslant 5 / 4$, so that $q \leqslant p / 5 \leqslant 1 / 5$. We may then take $z=q \sqrt{n}$, and we have

$$
P\left[\mu_{n}\left(A^{h}\right) \leqslant p / c\right] \leqslant P[|X| \geqslant q \cdot n] \leqslant 2 e^{-q^{2} n / 4} .
$$


Then the probability that $\mu_{n}\left(A^{h}\right) \leqslant p / c$ for some $\sigma \subseteq[0, J)$ such that $|\sigma|=s$ and some $h \in 2^{\sigma}$ is at most $\left(J^{s} / s !\right) 2^{s+1} e^{-q^{2} n / 4}$. The probability that this happens for some $s \leqslant k$ is at most $f(J)=e^{k} J^{k} e^{-r^{2} n / 4}$ where $r=2^{-k}\left(1-c^{-1}\right)$. Letting $J=\left[e^{n / K}\right]$, we have $f(J)=e^{k+n\left(k / K-r^{2} / 4\right)}$. Taking $K$ so that $k / K<r^{2} / 4$, we see that for sufficiently large $n$ we have $f(J)<1$. Therefore with positive probability, $\left\langle A_{i}: i<J\right\rangle$ is a $(c, k)$ independent sequence in $\Omega_{n}$.

Problem 2.4 Let $(\Omega, L(\mathcal{A}), L(\mu))$ be a uniform hyperfinite Loeb probability space with $|\Omega|=H$. By above lemma, for each infinite hyperinteger $K$ there exists an internal sequence $\left\langle A_{i}: i<\left[2^{H / K}\right]\right\rangle$ of internal subsets of $\Omega$ such that $L(\mu)\left(A_{i}\right)=\frac{1}{2}$ for every $i<\left[2^{H / K}\right]$ and the sequence is an independent sequence in $(\Omega, L(\mathcal{A}), L(\mu))$. Could the infinite hyperinteger $K$ above be replaced by a sufficiently large finite $k \in \mathbb{N}$ ?

Proof of Theorem 2.1: Let $H=|\Omega|$. Since $(\Omega, L(\mathcal{A}), L(\mu))$ is homogeneous we need only show that $\tau(\Omega)=\operatorname{card}\left(2^{H}\right)$. Clearly,

$$
\tau(\Omega) \leqslant \operatorname{card}(\mathcal{A})=\operatorname{card}\left(2^{H}\right)
$$

because for every $B \in L(\mathcal{A})$ there exists an $A \in \mathcal{A}$ with $\mathrm{L}(\mu)(A \Delta B)=0$. We need to show that $\tau(\Omega) \geqslant \operatorname{card}\left(2^{H}\right)$.

By Lemma 2.3 there is a finite $K \in \mathbb{N}$ such that there exists an internal $(2,2)$ independent sequence $\left\langle A_{i}: i<\left[2^{H / K}\right]\right\rangle$ of internal subsets of $\Omega$. Since $K$ is finite, one has

$$
\operatorname{card}\left(2^{H / K}\right)=\operatorname{card}\left(2^{H}\right)
$$

Suppose $\tau(\Omega)<\operatorname{card}\left(2^{H}\right)$. Take an $X \subseteq \mathcal{A}$ which completely generates $L(\mathcal{A})$ such that $\operatorname{card}(X)=\tau(\Omega)<\operatorname{card}\left(2^{H}\right)$. For each $i<\left[2^{H / K}\right]$ let $X_{i} \in X$ be such that $L(\mu)\left(A_{i} \Delta X_{i}\right)<\frac{1}{8}$. Clearly, there exist $i \neq j$ such that $X_{i}=X_{j}$. So

$$
L(\mu)\left(A_{i} \Delta A_{j}\right)=L(\mu)\left(A_{i} \cap\left(\Omega \backslash A_{j}\right)\right)+L(\mu)\left(\left(\Omega \backslash A_{i}\right) \cap A_{j}\right) \geqslant \frac{1}{4} .
$$

But on the other hand,

$$
L(\mu)\left(A_{i} \Delta A_{j}\right) \leqslant L(\mu)\left(A_{i} \Delta X_{i}\right)+L(\mu)\left(A_{j} \Delta X_{j}\right)<\frac{1}{4} .
$$

This contradiction completes the proof.

Remarks: (1) It is easy to see that if $L(\mu)(\Omega)=1$ is replaced $L(\mu)(\Omega)=r$ for any positive $r \in \mathbb{R}$, then Theorem 2.1 is still true. 
(2) If $\mu$ is not uniform but

$$
\max \{\mu(\{x\}): x \in \Omega\} \leqslant c \cdot \min \{\mu(\{x\}): x \in \Omega\}
$$

for some positive $c \in \mathbb{R}$, then it is also easy to see that Theorem 2.1 is true.

Next we apply Theorem 2.1 to a problem on the relation between the measure algebra automorphisms and point-automorphisms of a uniform hyperfinite Loeb space $(\Omega, L(\mathcal{A}), L(\mu))$. We will not give the relevant definitions involved. The reader can find them in [16], [17], [5] or [6].

Every point-automorphism induces a unique measure algebra automorphism. In [16] it is proved that if the nonstandard universe ${ }^{*} V$ is fully saturated, then every measure algebra automorphism of a uniform hyperfinite Loeb probability space is induced by a point-automorphism. Recently, the result was improved in [7], where full saturation is replaced by a weaker property called the $\aleph_{1}$-special model axiom. With the knowledge of the exact value of the Maharam spectrum of a uniform hyperfinite Loeb probability space we can show that some assumptions about the nonstandard universe in addition to $\omega_{1}$-saturation are needed to guarantee the truth of above result.

Corollary 2.5 Assume GCH. There is a nonstandard universe ${ }^{*} V$ in which there exists a uniform hyperfinite Loeb probability space $(\Omega, L(\mathcal{A}), L(\mu))$ such that the set of measure algebra automorphisms of $(\Omega, L(\mathcal{A}), L(\mu))$ has larger cardinality than the set of point-automorphisms of $(\Omega, L(\mathcal{A}), L(\mu))$.

Proof: Let $V$ be the original superstructure and let $\kappa>\operatorname{card}(V)$ be a regular cardinal. Then there is a $\kappa$-saturated nonstandard universe ${ }^{*} V$ in which there is an infinite hyperinteger $H$ such that $\operatorname{card}(H)=\kappa$ and $\operatorname{card}\left(2^{H}\right)=\kappa^{+}$(see [15], for example). Let $\Omega=[0, H)$ and let $(\Omega, L(\mathcal{A}), L(\mu))$ be the uniform hyperfinite Loeb probability space. The by Maharam's Theorem one can easily see that there are $2^{\kappa^{+}}=\kappa^{++}$distinct measure algebra automorphisms of $(\Omega, L(\mathcal{A}), L(\mu))$. One the other hand, there are at most $2^{\kappa}=\kappa^{+}$point-automorphisms because each pointautomorphism is a mapping from $\Omega$ to $\Omega$. 


\section{General hyperfinite Loeb spaces}

In this section we apply Theorem 2.1 to characterize the Maharam spectrum of an arbitrary atomless hyperfinite Loeb probability space $(\Omega, L(\mathcal{A}), L(\mu))$. Without loss of generality we assume that $\Omega=[0, H)$ and $\mathcal{A}$ is the set of all internal subsets of $\Omega$. Each point $x \in \Omega$ has weight $w(x)=\mu(\{x\})$. We may further assume without loss of generality that all weights are positive and that the elements of $\Omega$ are listed in order of decreasing weight, that is, $w(x) \geqslant w(y)>0$ whenever $x, y \in \Omega$ and $x \leqslant y$. Since we consider only atomless Loeb spaces, $w(x)$ is infinitesimal for every $x \in \Omega$. We associate with each $x \in \Omega$ the infinite cardinal $\kappa(x)=\operatorname{card}\left(2^{1 / w(x)}\right)$. Note that $\kappa(x) \leqslant \kappa(y)$ whenever $x \leqslant y$. Let

$$
\lambda_{\Omega}=(\bigcup\{\kappa(x): x \in \Omega\})^{+} .
$$

For each $\lambda<\lambda_{\Omega}$ let's define

$$
\mathcal{K}(\lambda)=\{x \in \Omega: \kappa(x)=\lambda\}, \quad \mathcal{K}(\leqslant \lambda)=\{x \in \Omega: \kappa(x) \leqslant \lambda\} .
$$

Clearly, $\mathcal{K}(\leqslant \lambda)$ is an initial segment of $\Omega$, and $\Omega=\bigcup_{\lambda<\lambda_{\Omega}} \mathcal{K}(\lambda)$.

Lemma 3.1 Every initial segment of $\Omega$ is $L(\mu)$-measurable.

Proof: Let $\mathcal{J}$ be any initial segment of $\Omega$. If $\mathcal{J}$ has a largest element $l$, then $\mathcal{J}=[0, l]$ is internal, so $\mathcal{J} \in L(\mathcal{A})$. Suppose $\mathcal{J}$ has no largest element. Let's define

$$
\underline{L}(\mu)(\mathcal{J})=\sup \{L(\mu)([0, x)): x \in \mathcal{J}\}
$$

and define

$$
\bar{L}(\mu)(\mathcal{J})=\inf \{L(\mu)([0, x)): x \in \Omega \backslash \mathcal{J}\} .
$$

If $\underline{L}(\mu)(\mathcal{J})=\bar{L}(\mu)(\mathcal{J})$, then $\mathcal{J} \in L(\mathcal{A})$ by the completeness of the Loeb measure. Suppose $\underline{L}(\mu)(\mathcal{J})=r<\bar{L}(\mu)(\mathcal{J})=s$. Let

$$
C=\left\{x \in \Omega: \mu([0, x)) \leqslant \frac{r+s}{2}\right\} .
$$

Then $C$ is internal, hence the largest element $c$ of $C$ exists. Using the fact that $w(c)$ and $w(c+1)$ are infinitesimals, it is easy to see that $c$ can belong to neither $\mathcal{J}$ nor $\Omega \backslash \mathcal{J}$. Hence we have a contradiction.

For each $\lambda<\lambda_{\Omega}$, the set $\mathcal{K}(\lambda)$ is a difference of two initial segments of $\Omega$. By above lemma, $\mathcal{K}(\lambda)$ is measurable. 
Theorem 3.2 The Maharam spectrum of an atomless hyperfinite Loeb probability space

$(\Omega, L(\mathcal{A}), L(\mu))$ is the set

$$
S=\left\{\lambda<\lambda_{\Omega}: L(\mu)(\mathcal{K}(\lambda))>0\right\}
$$

Proof: Let $\delta$ be the order type of $S$, let $\left\{\lambda_{\alpha}: \alpha<\delta\right\}$ be the increasing enumeration of $S$, and let $r_{\alpha}=L(\mu)\left(\mathcal{K}\left(\lambda_{\alpha}\right)\right)$. Since the sets $\mathcal{K}\left(\lambda_{\alpha}\right), \alpha<\delta$, are disjoint sets of positive Loeb measure, $\delta$ is an at most countable ordinal. Moreover, each $r_{\alpha}$ is positive and $\sum_{\alpha<\delta} r_{\alpha} \leqslant L(\mu)(\Omega)=1$.

Claim 3.2.1 $\sum_{\alpha<\delta} r_{\alpha}=1$.

Proof of Claim: Suppose $\sum_{\alpha<\delta} r_{\alpha}<1$. Let

$$
X=\Omega \backslash\left(\bigcup_{\alpha<\delta}\left(\mathcal{K}\left(\lambda_{\alpha}\right)\right)\right.
$$

Then $L(\mu)(X)=1-\sum_{\alpha<\delta} r_{\alpha}>0$. Let

$$
\kappa_{0}=\min \{\lambda: L(\mu)(X \cap \mathcal{K}(\leqslant \lambda))>0\} .
$$

Then $L(\mu)\left(X \cap \mathcal{K}\left(\leqslant \kappa_{0}\right)\right)>0$, and $L(\mu)(X \cap \mathcal{K}(\kappa))=0$ for each $\kappa<\kappa_{0}$. Let $\mathcal{J}$ be the initial segment $\mathcal{J}=\bigcup_{\kappa<\kappa_{0}} \mathcal{K}(\kappa)=\mathcal{K}\left(\leqslant \kappa_{0}\right) \backslash \mathcal{K}\left(\kappa_{0}\right)$. Since $\mathcal{J}$ is Loeb measurable,

$$
L(\mu)(\mathcal{J})=\underline{L}(\mu)(\mathcal{J})=\sup _{x \in \mathcal{J}} L(\mu)[0, x) .
$$

Therefore

$$
L(\mu)(X \cap \mathcal{J})=\sup _{x \in \mathcal{J}} L(\mu)(X \cap[0, x))=0,
$$

so $L(\mu)\left(X \cap \mathcal{K}\left(\kappa_{0}\right)\right)>0$. But then $\kappa_{0}=\lambda_{\alpha}$ for some $\alpha$. This contradicts the definition of $X \quad \square($ Claim3.2.1)

Let $\alpha<\delta$. Since $\mathcal{K}\left(\lambda_{\alpha}\right)$ is a convex subset of $\Omega$ of Loeb measure $r_{\alpha}$, there is a countable increasing chain of intervals

$$
I_{\alpha, n}=\left[x_{\alpha, n}, y_{\alpha, n}\right) \subseteq \mathcal{K}\left(\lambda_{\alpha}\right)
$$

such that

$$
\lim _{n \rightarrow \infty} L(\mu)\left(I_{\alpha, n}\right)=r_{\alpha}
$$


Then $L(\mu)\left(J_{\alpha}\right)=r_{\alpha}$ where $J_{\alpha}=\bigcup_{n \in \mathbb{N}} I_{\alpha, n}$. Now $\left\{J_{\alpha}: \alpha<\delta\right\}$ is a disjoint family of measurable subsets of $\Omega$ and

$$
L(\mu)\left(\bigcup_{\alpha<\delta} J_{\alpha}\right)=\sum_{\alpha<\delta} r_{\alpha}=1 .
$$

To prove the theorem we need only show that for each $\alpha<\delta$, we have $\tau(C)=\lambda_{\alpha}$ for every set $C \subseteq J_{\alpha}$ of positive Loeb measure.

Claim 3.2.2 Suppose that $\tau(B)=\lambda_{\alpha}$ for each $n \in \mathbb{N}$ and each $B \subseteq I_{\alpha, n}$ of positive Loeb measure. Then $\tau(A)=\lambda_{\alpha}$ for each $C \subseteq J_{\alpha}$ of positive Loeb measure.

Proof of Claim 3.2.2: For convenience we suppress the subscript $\alpha$ in the proof. For any $C \subseteq J$ with $L(\mu)(C)>0$ there is an $n \in \mathbb{N}$ such that $L(\mu)\left(C \cap I_{n}\right)>0$. Hence

$$
\tau(C) \geqslant \tau\left(C \cap I_{n}\right)=\lambda .
$$

So it suffices to show $\tau(J) \leqslant \lambda$. For each $n \in \mathbb{N}$, let $X_{n}$ be a set of cardinality $\lambda$ which completely generates $L(\mathcal{A}) \cap \mathcal{P}\left(I_{n}\right)$. Let $X=\bigcup_{n \in \mathbb{N}} X_{n}$. Then $\operatorname{card}(X)=\lambda$. We want to show that $X$ completely generates $L(\mathcal{A}) \cap \mathcal{P}(J)$. Given any measurable set $A \subseteq J$ and any positive $\epsilon \in \mathbb{R}$, there is an $n \in \mathbb{N}$ and a $B \in X_{n}$ such that

$$
L(\mu)\left(A \backslash I_{n}\right)<\frac{\epsilon}{2} \text { and } L(\mu)\left(\left(I_{n} \cap A\right) \Delta B\right)<\frac{\epsilon}{2} .
$$

Hence $L(\mu)(A \Delta B)<\epsilon$. This shows $X$ completely generates $L(\mathcal{A}) \cap \mathcal{P}(J)$, so $\tau(J) \leqslant \lambda$. $\square($ Claim 3.2.2)

By the claim above, the theorem is reduced to the following claim. Let's fix an $\alpha<\delta$ and an $n \in \mathbb{N}$.

Claim 3.2.3 For every $B \subseteq I_{\alpha, n}$ of positive Loeb measure, $\tau(B)=\lambda_{\alpha}$.

Proof of Claim 3.2.3: Let's again suppress the subscripts $\alpha$ and $n$ in the following proof. Without loss of generality, $0<L(\mu)(I)$, so $0<s t(\mu(I))$. We show first that $\tau(I) \leqslant \lambda$. Recall that $I=[x, y)$, where $x, y \in \mathcal{K}\left(\lambda_{\alpha}\right)$. Let $t=w(y)$. Then $w(z) \geqslant t$ for all $z \in I$, and $\operatorname{card}\left(2^{1 / t}\right)=\lambda$. By "splitting each point of $I$ into pieces", we can form a new hyperfinite measure space $\left(\Omega_{1}, \mathcal{A}_{1}, \mu_{1}\right)$ with weight function $w_{1}(u)$ such that $\Omega_{1}$ has an internal partition $\Omega_{1}=\bigcup_{z \in I} Y_{z}$ with

$$
\mu_{1}\left(Y_{z}\right)=w(z) \text { for each } z \in I
$$

and

$$
t \leqslant w_{1}(u) \leqslant 2 t \text { for each } u \in \Omega_{1} .
$$


By the remarks right after Theorem 2.1 one has that $\tau\left(\Omega_{1}\right)=\operatorname{card}\left(2^{\left|\Omega_{1}\right|}\right)$. Summing over $\Omega_{1}$,

$$
t \cdot\left|\Omega_{1}\right| \leqslant \sum_{u \in \Omega_{1}} w_{1}(u)=\mu(I) \leqslant 2 \cdot t \cdot\left|\Omega_{1}\right|
$$

which implies

$$
\frac{\mu(I)}{2 \cdot t} \leqslant\left|\Omega_{1}\right| \leqslant \frac{\mu(I)}{t} .
$$

Since $\operatorname{card}\left(2^{1 / t}\right)=\lambda$, we have $\operatorname{card}\left(2^{a / t}\right)=\lambda$ whenever $0<\operatorname{st}(a) \leqslant 1$. It follows that

$$
\lambda=\operatorname{card}\left(2^{\mu(I) / 2 t}\right) \leqslant \operatorname{card}\left(2^{\left|\Omega_{1}\right|}\right) \leqslant \operatorname{card}\left(2^{\mu(I) / t}\right)=\lambda .
$$

Hence $\tau\left(\Omega_{1}\right)=\lambda$.

Subclaim 3.2.3.1 $\tau(I) \leqslant \tau\left(\Omega_{1}\right)=\lambda$.

Proof of Subclaim 3.2.3.1: Take a set $X \subseteq \mathcal{A}_{1}$ of cardinality $\lambda$ which completely generates $L\left(\mathcal{A}_{1}\right)$. For each $A \in X$ define $C_{A} \subseteq I$ by

$$
C_{A}=\left\{z \in I: \mu_{1}\left(Y_{z} \cap A\right) \geqslant \frac{1}{2} \cdot w(z)\right\}
$$

Then

$$
I \backslash C_{A}=\left\{z \in I: \mu_{1}\left(Y_{z} \backslash A\right)>\frac{1}{2} \cdot w(z)\right\} .
$$

We want to show that $\left\{C_{A}: A \in X\right\}$ completely generates $L(\mathcal{A}) \cap \mathcal{P}(I)$. Given any internal $B \subseteq I$, let $D_{B}=\bigcup_{z \in B} Y_{z}$. Given any positive $\epsilon \in \mathbb{R}$, we need to find an $A \in X$ such that $\mu\left(C_{A} \Delta B\right)<\epsilon$. Let $A \in X$ be such that $\mu_{1}\left(A \Delta D_{B}\right)<\frac{\epsilon}{2}$. Then

$$
\begin{gathered}
\mu\left(C_{A} \Delta B\right)=\sum_{z \in C_{A} \backslash B} w(z)+\sum_{z \in B \backslash C_{A}} w(z) \\
\leqslant \sum_{z \in C_{A} \backslash B} 2 \mu_{1}\left(Y_{z} \cap A\right)+\sum_{z \in B \backslash C_{A}} 2 \mu_{1}\left(Y_{z} \backslash A\right) \\
\leqslant 2 \mu_{1}\left(A \backslash D_{B}\right)+2 \mu_{1}\left(D_{B} \backslash A\right)=2 \mu_{1}\left(A \Delta D_{B}\right)<\epsilon .
\end{gathered}
$$

Hence $\tau(I) \leqslant \operatorname{card}(X)=\lambda . \quad \square($ Subclaim 3.2.3.1)

We now show that for any internal $A \subseteq I$ with $L(\mu)(A)>0$, one has $\tau(A) \geqslant \lambda$. Let $s=w(x)$. Then $s \geqslant w(z)$ for every $z \in A$, and $\operatorname{card}\left(2^{1 / s}\right)=\lambda$. By "gluing points of $A$ together", we can a hyperfinite measure space $\left(\Omega_{2}, \mathcal{A}_{2}, \mu_{2}\right)$ with weight function $w_{2}$ where $\Omega_{2}$ is an internal partition of $A$ such that for each $Y \in \Omega_{2}$

$$
s \leqslant w_{2}(Y)=\mu(Y) \leqslant 2 s .
$$


As in the proof of Subclaim 3.2.3.2 one can show that

$$
\tau\left(\Omega_{2}\right)=\operatorname{card}\left(2^{1 / s}\right)=\lambda
$$

and

$$
\tau(A) \geqslant \tau\left(\Omega_{2}\right) .
$$

This completes the proof of the theorem.

From Theorem 3.2 one can see that the Maharam spectrum of a nonuniform hyperfinite Loeb probability space can be complex. In fact, for any countable set of infinite cardinals satisfying a minor condition one can choose an $\omega_{1}$-saturated nonstandard universe ${ }^{*} V$ and an atomless hyperfinite Loeb probability space in ${ }^{*} V$ such that the Maharam spectrum of the Loeb space is the prescribed set. Given a nonstandard universe ${ }^{*} V$, let

$$
D^{*} V=\left\{\operatorname{card}(H): H \text { is an infinite hyperinteger in }{ }^{*} V\right\} .
$$

For the existence of the nonstandard universe mentioned above we cite a result from $[13]$.

Suppose $D$ is a set of infinite cardinals satisfying

(1) $\lambda^{\aleph_{0}}=\lambda$ for each $\lambda \in D$, and

(2) If $\left\langle\kappa_{n}: n \in \mathbb{N}\right\rangle$ is a strictly increasing sequence in $D$, then $\left(\bigcup_{n \in \mathbb{N}} \kappa_{n}\right)^{+} \in D$.

Then there is a nonstandard universe ${ }^{*} V$, obtained by taking an ultrapower of the original superstructure, such that $D=D^{*} V$.

Note that any nonstandard universe obtained by an ultrapower construction is $\omega_{1}$-saturated, and if $H$ is an infinite hyperinteger in some $\omega_{1}$-saturated nonstandard universe, then $(\operatorname{card}(H))^{\aleph_{0}}=\operatorname{card}(H)$.

Theorem 3.3 Given any countable set of infinite cardinals $S$ and indexed set of positive real numbers $\left\{r_{\lambda}: \lambda \in S\right\}$ such that $\lambda^{\aleph_{0}}=\lambda$ for each $\lambda \in S$ and $\sum_{\lambda \in S} r_{\lambda}=1$, there is an $\omega_{1}$-saturated nonstandard universe ${ }^{*} V$ which has an atomless hyperfinite Loeb probability space $(\Omega, L(\mathcal{A}), L(\mu))$ with a Maharam partition $\left\{A_{\lambda}: \lambda \in S\right\}$ such that for each $\lambda \in S, L(\mu)\left(A_{\lambda}\right)=r_{\lambda}$ and $\tau\left(A_{\lambda}\right)=\lambda$. Hence $S$ is the Maharam spectrum of $(\Omega, L(\mathcal{A}), L(\mu))$. 
Proof: $\quad$ Let ${ }^{*} V$ be an $\omega_{1}$-saturated nonstandard universe such that $S \subseteq D^{*} V$. The existence of such nonstandard universe is guaranteed by the result in [13] mentioned above. Note that if $\operatorname{card}(K)=\lambda$ and $H=\left[\log _{2} K\right]$, then $\operatorname{card}\left(2^{H}\right)=\lambda$. Hence for each $\lambda \in S$ there is an infinite hyperinteger $H_{\lambda}$ such that $\operatorname{card}\left(2^{H_{\lambda}}\right)=\lambda$. Let

$$
S=\left\{\lambda_{n}: n<k\right\}
$$

be an enumeration, where $k$ can be either finite or $\omega$. Let $K_{-1}=0$ and for each $n<k$ let $K_{n}=K_{n-1}+H_{\lambda_{n}}$. Let $W$ be the function on $\bigcup_{n<k}\left[0, K_{n}\right)$ such that

$$
W(z)=r_{\lambda_{n}} / H_{\lambda_{n}}
$$

for each $n<k$ and $z \in\left[K_{n-1}, K_{n}\right)$. By $\omega_{1}$-saturation one can find a $K \in{ }^{*} \mathbb{N}$ such that $K \geqslant K_{n}$ for each $n<k$, and extend $W$ to an internal function $W:[0, K) \rightarrow{ }^{*}[0,1]$ such that $\sum_{z \in[0, K)} W(z) \leqslant 1$. Let $\Omega=[0, K)$ and let $\mu$ be the measure on ${ }^{*} \mathcal{P}(\Omega)$ with weight function $W$. It is easy to see that $\left(\Omega, L\left({ }^{*} \mathcal{P}(\Omega)\right), L(\mu)\right)$ is the desired space, because

$$
L(\mu)\left(\Omega \backslash \bigcup_{n<k}\left[0, K_{n}\right)\right)=0
$$

\section{Loeb spaces generated by standard spaces}

In this section we characterize the Maharam spectrum of a Loeb probability space $\left({ }^{*} \Omega, L\left({ }^{*} \mathcal{B}\right), L\left({ }^{*} \nu\right)\right)$ generated by a standard probability space $(\Omega, \mathcal{B}, \nu)$ in $V$. Note that if $X$ and $Y$ are two sets in $V$ with the same cardinality, then ${ }^{*} X$ and ${ }^{*} Y$ in ${ }^{*} V$ have the same external cardinality because if $i$ is a bijection from $X$ to $Y$, then ${ }^{*} i$ is a bijection from ${ }^{*} X$ to ${ }^{*} Y$.

Theorem 4.1 If a standard probability space $(\Omega, \mathcal{B}, \nu)$ is homogeneous and has $M a$ haram type $\kappa$, then the Loeb probability space $\left({ }^{*} \Omega, L\left({ }^{*} \mathcal{B}\right), L\left({ }^{*} \nu\right)\right)$ generated by $(\Omega, \mathcal{B}, \nu)$ is also homogeneous and has Maharam type $\operatorname{card}\left({ }^{*} \kappa\right)$.

Proof: First we show that the Loeb space $\left({ }^{*} \Omega, L\left({ }^{*} \mathcal{B}\right), L\left({ }^{*} \nu\right)\right)$ has Maharam type $\tau\left({ }^{*} \Omega\right) \leqslant \operatorname{card}\left({ }^{*} \kappa\right)$. We will then show that for each $A \in L\left({ }^{*} \mathcal{B}\right)$ with $L\left({ }^{*} \nu\right)(A)>0$, one has $\tau(A) \geqslant \operatorname{card}\left({ }^{*} \kappa\right)$. Without loss of generality we may take the set $Z$ of urelements to contain $\kappa$. 
Suppose $X \subseteq \mathcal{B}$ is a set of cardinality $\kappa$ which completely generates $\mathcal{B}$. By Maharam's Theorem, $(\Omega, \mathcal{B}, \nu)$ is measure algebra isomorphic to the space $\left(\{0,1\}^{\kappa}\right)$ defined in the introduction. Hence there is an independent sequence $\left\langle B_{\alpha}: \alpha<\kappa\right\rangle$ of measurable subsets of $\Omega$ such that $\nu\left(B_{\alpha}\right)=\frac{1}{2}$. For each $n \in \mathbb{N}$ let $\mathcal{F}_{n}$ be a maximal family of measurable subsets of $\Omega$ containing all $B_{\alpha}$ 's such that $\nu(F \Delta G) \geqslant \frac{1}{n}$ for any two different $F$ and $G$ in $\mathcal{F}_{n}$. Clearly, $\operatorname{card}\left(\mathcal{F}_{n}\right) \geqslant \kappa$ for each $n>1$.

Claim 4.1.1 $\operatorname{card}\left(\mathcal{F}_{n}\right)=\kappa$ for each $n>1$.

Proof of Claim 4.1.1: Suppose $\operatorname{card}\left(\mathcal{F}_{n}\right)>\kappa$. For each $F \in \mathcal{F}_{n}$ there exists an $x_{F} \in X$ such that $\nu\left(F \Delta x_{F}\right)<\frac{1}{2 n}$. Since $\operatorname{card}\left(\mathcal{F}_{n}\right)>\operatorname{card}(X)$, there exist at least two distinct elements, $F$ and $G$ in $\mathcal{F}_{n}$, such that $x_{F}=x_{G}$. Hence

$$
\nu(F \Delta G) \leqslant \nu\left(F \Delta x_{F}\right)+\nu\left(x_{G} \Delta G\right)<\frac{1}{n},
$$

which contradicts $\nu(F \Delta G) \geqslant \frac{1}{n} . \quad \square($ Claim 4.1.1)

Now let $\mathcal{F}=\bigcup_{n \in \mathbb{N}}{ }^{*} \mathcal{F}_{n}$. Then one has $\operatorname{card}\left({ }^{*} \mathcal{F}_{n}\right)=\operatorname{card}\left({ }^{*} \kappa\right)$ for each $n>1$, and

$$
\operatorname{card}(\mathcal{F})=\aleph_{0} \cdot \operatorname{card}\left({ }^{*} \kappa\right)=\operatorname{card}\left({ }^{*} \kappa\right) .
$$

Claim 4.1.2 $\mathcal{F}$ completely generates $L\left({ }^{*} \mathcal{B}\right)$.

Proof of Claim 4.1.2 Let $n \in \mathbb{N}$. Since the sentence

$$
\text { " }(\forall B \in \mathcal{B})\left(\exists F \in \mathcal{F}_{n}\right)\left(\nu(B \Delta F)<\frac{1}{n}\right) "
$$

is true by the maximality of $\mathcal{F}_{n}$, then by the Transfer Principle the sentence

$$
\text { " }\left(\forall B \in{ }^{*} \mathcal{B}\right)\left(\exists F \in{ }^{*} \mathcal{F}_{n}\right)\left({ }^{*} \nu(B \Delta F)<\frac{1}{n}\right) "
$$

is also true in ${ }^{*} V$. Hence for every $A \in{ }^{*} \mathcal{B}$ and every positive $\epsilon \in \mathbb{R}$ there exists an $F \in \mathcal{F}$ such that ${ }^{*} \nu(A \Delta F)<\epsilon$. This ends the proof. $\square($ Claim 4.1.2)

By Claim 4.1.2 we have that $\tau\left({ }^{*} \Omega\right) \leqslant \operatorname{card}\left({ }^{*} \kappa\right)$. Let $C \in{ }^{*} \mathcal{B}$ with

$$
L\left({ }^{*} \nu\right)(C)=r>0 .
$$

Claim 4.1.3 $\tau(C) \geqslant \operatorname{card}\left({ }^{*} \kappa\right)$.

Proof of Claim 4.1.3: Suppose

$$
\lambda=\tau(C)<\operatorname{card}\left({ }^{*} \kappa\right) .
$$


Let $X$ be a subset of ${ }^{*} \mathcal{B}$ of cardinality $\lambda$ which completely generates the restriction $L\left({ }^{*} \mathcal{B}\right) \cap \mathcal{P}(C)$. Let $\mathcal{D}=\left\langle B_{\alpha}: \alpha<\kappa\right\rangle$ be the independent sequence of measurable subsets of $\Omega$ such that $\nu\left(B_{\alpha}\right)=\frac{1}{2}$ for each $\alpha<\kappa$. Then ${ }^{*} \mathcal{D}=\left\langle{ }^{*} B_{\alpha}: \alpha<{ }^{*} \kappa\right\rangle$ is an independent sequence with ${ }^{*} \nu\left({ }^{*} B_{\alpha}\right)=\frac{1}{2}$ for each $\alpha<{ }^{*} \kappa$ in $\left({ }^{*} \Omega, L\left({ }^{*} \mathcal{B}\right), L\left({ }^{*} \nu\right)\right)$. Choose $m \in \mathbb{N}$ first and then choose $n \in \mathbb{N}$ large enough such that

$$
\frac{1}{2^{m}}<\frac{r}{2}-\frac{m}{n} .
$$

For each $\alpha<{ }^{*} \kappa$ let $x_{\alpha} \in X$ be such that

$$
{ }^{*} \nu\left(\left(A \cap{ }^{*} B_{\alpha}\right) \Delta x_{\alpha}\right)<\frac{1}{n} .
$$

Since $\operatorname{card}\left({ }^{*} \kappa\right)>\operatorname{card}(X)$, there exists an infinite $E \subseteq{ }^{*} \kappa$ and an $x \in X$ such that for each $\alpha \in E$ one has $x_{\alpha}=x$. Suppose ${ }^{*} \nu(x) \geqslant \frac{r}{2}$ (otherwise replace $x$ by $C \backslash x$ and replace ${ }^{*} B_{\alpha}$ by $\left.{ }^{*} \Omega \backslash{ }^{*} B_{\alpha}\right)$. Then for $\alpha_{1}<\alpha_{2}<\ldots<\alpha_{m}$ in $E$ one has

$$
{ }^{*} \nu\left(\bigcap_{i=1}^{m}{ }^{*} B_{\alpha_{i}} \cap C \cap x\right) \leqslant{ }^{*} \nu\left(\bigcap_{i=1}^{m}{ }^{*} B_{\alpha_{i}}\right) \leqslant \frac{1}{2^{m}} .
$$

On the other hand, one has

$$
\bigcap_{i=1}^{m} B^{*} B_{\alpha_{i}} \cap C \cap x=C \cap x \backslash\left(\bigcup_{i=1}^{m}\left(x \backslash{ }^{*} B_{\alpha_{i}}\right)\right) .
$$

Hence

$$
{ }^{*} \nu\left(\bigcap_{i=1}^{m}{ }^{*} B_{\alpha_{i}} \cap C \cap x\right) \geqslant \frac{r}{2}-\sum_{i=1}^{m}{ }^{*} \nu\left(x \backslash{ }^{*} B_{\alpha_{i}}\right) \geqslant \frac{r}{2}-\frac{m}{n},
$$

which contradicts the choice of $m$ and $n$.

Theorem 4.2 Let $S$ be the Maharam spectrum of a standard probability space $(\Omega, \mathcal{B}, \nu)$ in $V$. The Loeb probability space $\left({ }^{*} \Omega, L\left({ }^{*} \mathcal{B}\right), L\left({ }^{*} \nu\right)\right)$ generated by $(\Omega, \mathcal{B}, \nu)$ has Maharam spectrum

$$
T=\left\{\operatorname{card}\left({ }^{*} \kappa\right): \kappa \in S\right\} .
$$

Proof: Let $P=\left\{C_{\kappa}: \kappa \in S\right\}$ be a partition of $\Omega$ and let $R=\left\{r_{\kappa}: \kappa \in S\right\}$ be a set of positive real numbers such that

$$
\nu\left(C_{\kappa}\right)=r_{\kappa}, \quad \sum_{\kappa \in S} r_{\kappa}=1,
$$


and $(\Omega, \mathcal{B}, \nu) \uparrow C_{\kappa}$ is homogeneous with Maharam type $\kappa$. By Theorem 4.1 we know that $\left({ }^{*} \Omega, L\left({ }^{*} \mathcal{B}\right), L\left({ }^{*} \nu\right)\right) \uparrow{ }^{*} C_{\kappa}$ is homogeneous with Maharam type $\operatorname{card}\left({ }^{*} \kappa\right)$, and $L\left({ }^{*} \nu\right)\left({ }^{*} C_{\kappa}\right)=r_{\kappa}$. Since

$$
\left\{{ }^{*} C_{\kappa}: \kappa \in S\right\}
$$

is a disjoint family, we have that

$$
L\left({ }^{*} \nu\right)\left({ }^{*} \Omega \backslash \bigcup_{\kappa \in S}^{*} C_{\kappa}\right)=0
$$

For each $\lambda \in T$ let

$$
D_{\lambda}=\bigcup\left\{{ }^{*} C_{\kappa}: \kappa \in S \text { and } \operatorname{card}\left({ }^{*} \kappa\right)=\lambda\right\}
$$

Then it is easy to see that $(\Omega, L(\mathcal{A}), L(\mu)) \uparrow D_{\lambda}$ is homogeneous and has Maharam type $\lambda$. Clearly, $D_{\lambda}$ and $D_{\lambda^{\prime}}$ are disjoint for different $\lambda$ and $\lambda^{\prime}$, and

$$
L\left({ }^{*} \nu\right)\left(\bigcup_{\lambda \in T} D_{\lambda}\right)=1 .
$$

So $T$ is the Maharam spectrum of $\left({ }^{*} \Omega, L\left({ }^{*} \mathcal{B}\right), L\left({ }^{*} \nu\right)\right)$.

\section{Hyperfinite approximation}

Throughout this section, we assume that $(\Omega, \mathcal{A}, \mu)$ is an internal (*countably additive) probability space.

Let us say that $(\Omega, \mathcal{C}, \mu)$ is a hyperfinite approximation of $(\Omega, \mathcal{A}, \mu)$ if $\mathcal{C}$ is a hyperfinite subalgebra of $\mathcal{A}$ and every Maharam partition of the Loeb space $(\Omega, L(\mathcal{C}), L(\mu))$ is also a Maharam partition of $(\Omega, L(\mathcal{A}), L(\mu))$ with the same Maharam types.

It follows that $(\Omega, L(\mathcal{A}), L(\mu))$ and $(\Omega, L(\mathcal{C}), L(\mu))$ have the same Maharam spectra.

In this section we will determine when a hyperfinite approximation of $(\Omega, \mathcal{A}, \mu)$ exists. It turns out that this happens if and only if the Maharam type of $(\Omega, L(\mathcal{A}), L(\mu))$ is not too large. The question is of interest because the results in $\S \S 2,3$ completely characterize the Maharam spectra of hyperfinite Loeb spaces, and as the next lemma shows, the Loeb space $(\Omega, L(\mathcal{C}), L(\mu))$ is essentially a hyperfinite Loeb space. 
Lemma 5.1 Let $(\Omega, \mathcal{C}, \mu)$ be an internal probability space such that the algebra of sets $\mathcal{C}$ is hyperfinite. Then the Loeb space $(\Omega, L(\mathcal{C}), L(\mu))$ has Maharam type $\leqslant \operatorname{card}(H)$ for some $H \in{ }^{*} \mathbb{N}$, and there is a measure algebra isomorphism between $(\Omega, L(\mathcal{C}), L(\mu))$ and a hyperfinite Loeb space.

Proof: For each $x \in \Omega$, let

$$
\hat{x}=\bigcap\{C \in \mathcal{C}: x \in C\}
$$

We can identify each $\hat{x}$ with a point. For each $C \in \mathcal{C}$ let $\hat{C}=\{\hat{x}: x \in C\}$. Let

$$
\hat{\Omega}=\{\hat{x}: x \in \Omega\} \quad \hat{\mathcal{C}}=\{\hat{C}: C \in \mathcal{C}\}
$$

and let $\hat{\mu}$ be the measure on $\hat{\mathcal{C}}$ induced by $\mu$. Then $(\hat{\Omega}, \hat{\mathcal{C}}, \hat{\mu})$ is hyperfinite, and the mapping $C \mapsto \hat{C}$ induces a measure algebra isomorphism between the corresponding Loeb spaces.

We now consider the case that $(\Omega, \mathcal{A}, \mu)$ is *atomless.

Lemma 5.2 Suppose $(\Omega, \mathcal{A}, \mu)$ is *atomless. Then the Loeb space $(\Omega, L(\mathcal{A}), L(\mu))$ has Maharam type $\geqslant \operatorname{card}\left({ }^{*} \mathbb{N}\right)$.

Proof: By the transfer principle, $\mathcal{A}$ contains an internal sequence of independent sets indexed by ${ }^{*} \mathbb{N}$.

Theorem 5.3 Suppose $(\Omega, \mathcal{A}, \mu)$ is *atomless and let $\lambda$ be the Maharam type of the Loeb space $(\Omega, L(\mathcal{A}), L(\mu))$.

If $\lambda=\operatorname{card}\left({ }^{*} \mathbb{N}\right)=\operatorname{card}(H)$ for some $H \in{ }^{*} \mathbb{N}$, then $(\Omega, \mathcal{A}, \mu)$ has a hyperfinite approximation and $(\Omega, L(\mathcal{A}), L(\mu))$ is homogeneous.

Otherwise (i.e., if either $\lambda>\operatorname{card}\left({ }^{*} \mathbb{N}\right)$ or $\operatorname{card}(H)<\operatorname{card}\left({ }^{*} \mathbb{N}\right)$ for all $\left.H \in{ }^{*} \mathbb{N}\right)^{4}$, the Maharam spectrum of $(\Omega, L(\mathcal{A}), L(\mu))$ is disjoint from the Maharam spectrum of $(\Omega, L(\mathcal{C}), L(\mu))$ for every hyperfinite $\mathcal{C} \subseteq \mathcal{A}$.

\section{Proof:}

The "otherwise" part follows from the preceding two lemmas.

\footnotetext{
${ }^{4}$ The nonstandard universes with $\operatorname{card}(H)<\operatorname{card}\left({ }^{*} \mathbb{N}\right)$ for every $H \in{ }^{*} \mathbb{N}$ are called $\operatorname{card}\left({ }^{*} \mathbb{N}\right)$ Archimedean. See [12] or [8] for the discussion of the existence of such nonstandard universes.
} 
Suppose that $\lambda=\operatorname{card}\left({ }^{*} \mathbb{N}\right)=\operatorname{card}(H)$ for some $H \in{ }^{*} \mathbb{N}$. For any $D \in \mathcal{A}$ with $L(\mu)(D)>0$ the measure space $(\Omega, \mathcal{A}, \mu) \mid D$ is still internal and *atomless. Hence

$$
\lambda \geqslant \tau(D) \geqslant \operatorname{card}\left({ }^{*} \mathbb{N}\right) \geqslant \lambda .
$$

Therefore $(\Omega, L(\mathcal{A}), L(\mu))$ is homogeneous.

By the Transfer Principle we can partition $\Omega$ into $H$ disjoint sets $\left\{A_{n}: n<H\right\}$ such that for each $n<H$ the set $A_{n}$ is in $\mathcal{A}$ and has $\mu$-measure $1 / H$. Let $\mathcal{C}$ be the internal subalgebra of $\mathcal{A}$ generated by $\left\{A_{n}: n<H\right\}$. Then $\mathcal{C}$ is hyperfinite. Clearly, $(\Omega, L(\mathcal{C}), L(\mu))$ is measure algebra isomorphic to a uniform hyperfinite Loeb probability space over a set with internal cardinality $H$. Hence $(\Omega, L(\mathcal{C}), L(\mu))$ is homogeneous and has Maharam type $\operatorname{card}\left(2^{H}\right)$. Since

$$
\lambda=\operatorname{card}(H) \leqslant \operatorname{card}\left(2^{H}\right) \leqslant \operatorname{card}\left({ }^{*} \mathbb{N}\right)=\lambda,
$$

$(\Omega, L(\mathcal{C}), L(\mu))$ has Maharam type $\lambda$ and is a hyperfinite approximation of $(\Omega, L(\mathcal{A}), L(\mu))$.

We now take up the case where $(\Omega, \mathcal{A}, \mu)$ is *atomistic.

Lemma 5.4 Suppose $\mathcal{C}$ is a hyperfinite subalgebra of $\mathcal{A}$ such that for each $A \in \mathcal{A}$ there is a $C \in \mathcal{C}$ with $L(\mu)(A \Delta C)=0$. Then $(\Omega, \mathcal{C}, \mu)$ is a hyperfinite approximation of $(\Omega, \mathcal{A}, \mu)$.

Proof: Straightforward.

Theorem 5.5 Suppose that $(\Omega, \mathcal{A}, \mu)$ is ${ }^{*}$ atomistic, or at least that every set of positive Loeb measure contains a ${ }^{*}$ atom of $(\Omega, \mathcal{A}, \mu)$. Then $(\Omega, \mathcal{A}, \mu)$ has a hyperfinite approximation.

Proof: By transfer, there is an internal maximal pairwise disjoint family $D$ of *atoms in $(\Omega, \mathcal{A}, \mu)$. Moreover, $D$ is hyperfinite or *countable, so its union belongs to $\mathcal{A}$. $\cup D$ has Loeb measure one, since its complement does not contain a *atom. By ${ }^{*}$ countable additivity, for each $\delta>0$ there is a hyperfinite $D_{0} \subseteq D$ such that $\mu\left(\cup D_{0}\right) \geqslant \mu(\bigcup D)-\delta$. Taking $\delta$ infinitesimal, we get a $D_{0}$ such that $\cup D_{0}$ has Loeb measure one. Let $\mathcal{C}$ be the ${ }^{*}$ subalgebra of $\mathcal{A}$ generated by $D_{0}$. Then $\mathcal{C}$ is hyperfinite. It is easily seen that $\mathcal{C}$ satisfies the hypotheses of Lemma 5.4.

Finally, we combine the two preceding theorems to take care of the general case. 
Theorem 5.6 The following are equivalent:

(i) $(\Omega, \mathcal{A}, \mu)$ has a hyperfinite approximation.

(ii) The corresponding Loeb space $(\Omega, L(\mathcal{A}), L(\mu))$ has Maharam type $\leqslant \operatorname{card}(H)$ for some $H \in{ }^{*} \mathbb{N}$.

Proof: Lemma 5.1 shows that (i) implies (ii). Assume (ii). By the Transfer Principle one can partition $\Omega$ into two sets $\Omega_{0}$ and $\Omega_{1}$ such that $\Omega_{0}$ is the union of an at most * countable family of *atoms, and $\Omega_{1}$ contains no *atoms. Without loss of generality we assume $L(\mu)\left(\Omega_{i}\right)>0$ for $i=0,1$ (otherwise the case is reduced to either Theorem 5.5 or Theorem 5.3). For $i=0,1$ let $\mathcal{A}_{i}$ and $\mu_{i}$ be the restrictions of $\mathcal{A}$ and $\mu$ to $\Omega_{i}$. By Theorems 5.5 and 5.3 , for $i=0,1,\left(\Omega_{i}, \mathcal{A}_{i}, \mu_{i}\right)$ has a hyperfinite approximation $\left(\Omega_{i}, \mathcal{C}_{i}, \mu_{i}\right)$. Now let $\mathcal{C}$ be the internal subalgebra of $\mathcal{A}$ generated by $\mathcal{C}_{0} \cup \mathcal{C}_{1}$. It is easy to see that $(\Omega, L(\mathcal{C}), L(\mu))$ is a hyperfinite approximation of $(\Omega, \mathcal{A}, \mu)$.

\section{Working in a nice nonstandard universe}

In this section we show that by working within a nice nonstandard universe or imposing a bound on the internal cardinality of the sample space $\Omega$, one can make the Maharam spectra of Loeb spaces simple.

Theorem 6.1 There exists an infinite hyperinteger $H$ such that every hyperfinite Loeb probability space $(\Omega, L(\mathcal{A}), L(\mu))$ with $|\Omega| \leqslant H$ is homogeneous and has Maharam type $\operatorname{card}(H)$.

Proof: Let $\lambda=\min \left\{\operatorname{card}(K): K \in{ }^{*} \mathbb{N} \backslash \mathbb{N}\right\}$ and let $H$ be an infinite hyperinteger such that $\operatorname{card}\left(2^{H}\right)=\lambda$. Then $\operatorname{card}(H)=\lambda$. Suppose $(\Omega, L(\mathcal{A}), L(\mu))$ is a hyperfinite Loeb probability space such that $|\Omega| \leqslant H$. For any $A \in \mathcal{A}$ with $L(\mu)(A)>0$ there is a sequence $\left\langle A_{n}: n \in \mathbb{N}\right\rangle$ of internal subsets of $A$ such that $L(\mu)\left(A_{n} \Delta A_{m}\right)>\frac{1}{4}$ for different $n, m \in \mathbb{N}$ because $(\Omega, L(\mathcal{A}), L(\mu))$ is atomless. By $\omega_{1}$-saturation the sequence can be extended to a hyperfinite internal sequence $\left\langle A_{n}: n<K\right\rangle$ for some infinite hyperinteger $K$ such that $L(\mu)\left(A_{n} \Delta A_{m}\right)>\frac{1}{4}$ for different $n, m<K$. So one has that

$$
\lambda \leqslant \operatorname{card}(K) \leqslant \tau(A) \leqslant \operatorname{card}\left({ }^{*} \mathcal{P}(\Omega)\right) \leqslant \operatorname{card}\left(2^{H}\right)=\lambda .
$$

Hence $(\Omega, L(\mathcal{A}), L(\mu))$ is homogeneous and has Maharam type $\operatorname{card}(H)$. 
Theorem 6.2 If there is a cardinal $\lambda$ such that $\lambda=\operatorname{card}(H)$ for each infinite hyperinteger $H$, then every hyperfinite Loeb probability space is homogeneous and has Maharam type $\lambda$.

Proof: Similar to the proof of Theorem 6.1.

Remark: If the nonstandard universe ${ }^{*} V$ is obtained from an ultrapower construction of the standard superstructure modulo an ultrafilter over a countable set, then $\operatorname{card}(H)=2^{\aleph_{0}}$ for every infinite hyperinteger $H$ in ${ }^{*} V$.

Theorem 6.3 If there is a cardinal $\lambda$ such that $\operatorname{card}(X)=\lambda$ for every infinite internal set $X$, then every Loeb probability space is homogeneous and has Maharam type $\lambda$.

Proof: Left to the reader.

Remark: If ${ }^{*} V$ satisfies the $\aleph_{1}$-isomorphism property or the $\aleph_{1}$-special model axiom (see [3], [17], [5] or [6] for the definitions), then all infinite internal sets have the same external cardinality.

\section{References}

[1] Chang, C. C. and Keisler, H. J., Model Theory, North-Holland, third edition 1990.

[2] Fremlin, D., Measure algebra, Chapter 22, Vol. 3 of the Handbook of Boolean Algebra, ed. by J. D. Monk with R. Bonnet, North-Holland 1989.

[3] Henson, C. W., The isomorphism property in nonstandard analysis and its use in the theory of Banach space, The Journal of Symbolic Logic, 39 (1974), 717-731.

[4] Henson, C. W., Foundations of nonstandard analysis-A gentle introduction to nonstandard extension, in Nonstandard Analysis: Theory and Applications, ed. by N. J. Cutland, C. W. Henson, and L. Arkeryd, Kluwer Academic Publishers 1997.

[5] Jin, R., The isomorphism property versus the special model axiom, The Journal of Symbolic Logic, 57 (1992), 975-987. 
[6] Jin, R., Better nonstandard universes with applications, in Nonstandard Analysis: Theory and Applications, ed. by N. J. Cutland, C. W. Henson, and L. Arkeryd, Kluwer Publishers 1997.

[7] Jin, R., Distinguishing three saturation properties in nonstandard analysis, Annals of Pure and Applied Logic, accepted.

[8] Jin, R. and Shelah, S., Possible size of an ultrapower of $\omega$, Archive for Mathematical Logic, 38 (1999), pp. 61-77.

[9] Jin, R. and Shelah, S., Compactness of Loeb spaces, The Journal of Symbolic Logic, 63 (1998), pp. 1371-1392.

[10] Keisler, H. J., An Infinitesimal Approach to Stochastic Analysis, A.M.S. Memoirs, 297 (1984).

[11] Keisler, H. J., Rich and saturated adapted spaces, Advances in Mathematics, 128 (1997), pp. 242-288.

[12] Keisler, H. J. and Schmerl, J. H., Making the hyperreal line both saturated and complete, The Journal of Symbolic Logic, 56 (1991), 1016-1025.

[13] Koppelberg, S., Cardinalities of ultraproducts of finite sets, The Journal of Symbolic Logic, 45 (1980), 574-584.

[14] Lindstrom, T., An invitation to nonstandard analysis, in Nonstandard Analysis and Its Application, ed. by N. Cutland, Cambridge University Press 1988.

[15] Miller, A. W., Set theoretic properties of Loeb measure, The Journal of Symbolic Logic, 55 (1990), 1022-1036.

[16] Ross, D. A., Automorphisms of the Loeb algebra, Fundamenta Mathematicae, 128 (1987), 29-36.

[17] Ross, D. A., The special model axiom in nonstandard analysis, The Journal of Symbolic Logic, 55 (1990), 1233-1242.

[18] Ross, D. A., Compact measures have Loeb preimages, Proceedings of American Mathematical Society, 115 (1992), 365-370. 
[19] Ross, D. A., Loeb Measure and Probability, in Nonstandard Analysis: Theory and Applications, ed. by N. J. Cutland, C. W. Henson, and L. Arkeryd, Kluwer Publishers 1997.

Department of Mathematics, College of Charleston

Charleston, SC 29424

e-mail: jin@math.cocf.edu

Department of Mathematics, University of Wisconsin

Madison, WI 53706

e-mail:keisler@math.wisc.edu 